\title{
A!
}

This is an electronic reprint of the original article.

This reprint may differ from the original in pagination and typographic detail.

Kallunki, J.; Tornikoski, M.; Oinaskallio, E.; Kirves, P.; Aatrokoski, J.; Mujunen, A.; Tammi, J.

\section{Solar observing system for radio frequencies 5-120 MHz}

Published in:

Astronomische Nachrichten

DOI:

10.1002/asna.201913545

Published: 01/11/2018

Document Version

Peer reviewed version

Please cite the original version:

Kallunki, J., Tornikoski, M., Oinaskallio, E., Kirves, P., Aatrokoski, J., Mujunen, A., \& Tammi, J. (2018). Solar observing system for radio frequencies 5-120 MHz. Astronomische Nachrichten, 339(9-10), 656-660.

https://doi.org/10.1002/asna.201913545

This material is protected by copyright and other intellectual property rights, and duplication or sale of all or part of any of the repository collections is not permitted, except that material may be duplicated by you for your research use or educational purposes in electronic or print form. You must obtain permission for any other use. Electronic or print copies may not be offered, whether for sale or otherwise to anyone who is not an authorised user. 
DOI: $\mathrm{xxx} / \mathrm{xxxx}$

\title{
ARTICLE TYPE
}

\section{Solar observing system for radio frequencies 5-120 MHz}

\author{
Juha Kallunki* | Merja Tornikoski | Erkki Oinaskallio | Petri Kirves I Juha Aatrokoski | Ari \\ Mujunen I Joni Tammi
}

${ }^{1}$ Metsähovi Radio Observatory, Aalto

University, Kylmälä, Finland

Correspondence

*Juha Kallunki Email: juha.kallunki@ aalto.fi

\begin{abstract}
Aalto University Metsähovi Radio Observatory (MRO) in Finland installed and started operating a new low frequency solar radio monitoring instrument in October 2017. The main component of the new system is a crossed dipole antenna, and it uses a Callisto spectrometer as backend. The operational range of the new radio telescope is between 5 and $120 \mathrm{MHz}$, and its main purpose is to detect solar radio bursts. The coverage of MRO solar radio observations now extends from $5 \mathrm{MHz}$ to $37 \mathrm{GHz}$, enabling studies of solar flares over a very wide frequency range. The first solar radio bursts were detected with the new system in March 2018. In this article we describe the new system and report the first flare observations.

KEYWORDS:

Long Wavelength Array (LWA), solar radio observations, Callisto, solar radio burst
\end{abstract}

\section{1 | INTRODUCTION}

A crossed dipole antenna (CDA) is ideal for solar radio burst observations due to its high sensitivity and frequency range that is suitable for monitoring Type II, III, and IV solar bursts. It is also very cost-effective. The antenna is constructed as an omni-directional dome shaped structure, so there is no need for a tracking system that would increase the complexity and cost of the system. Additionally, CDA has a good weather survivability, especially against strong wind. The need for maintenance is also very low.

Aalto University Metsähovi Radio Observatory 11 (MRO), located in southern Finland (GPS coordinates: N 60:13.04, E 24:23.35), installed a CDA manufactured by Reeve Observatory (Reeve, 2018) in October 2017. The new antenna, dubbed the Metsähovi Solar Observing Low-frequency Antenna METSOLA, has frequency coverage between 5 and $120 \mathrm{MHz}$, and it expands MRO's solar radio observing coverage Kallunki et al., 2018) to low frequencies. The low frequency solar radio observations are also conducted with The LOw Frequency ARray (LOFAR) radio interferometer. It is able to

\footnotetext{
${ }^{1}$ Homepage: http://www.aalto.fi/metsahovi-radio-observatory
}

observe solar radio bursts at low frequencies: $10-90 \mathrm{MHz}$ and 110-250 MHz (Mann et al., 2018).

The backend is a Callisto spectrometer (Callisto, 2018), designed to observe the dynamic spectra of solar flares and widely used for solar monitoring at frequencies below $1000 \mathrm{MHz}$. METSOLA operates in the frequency range of classic solar radio bursts Types II, III and IV. At this frequency range it is possible to study properties of bursts in the solar corona. At these frequencies the most prevailing emission mechanism is plasma emission. The new instrument enables studies of a versatile nature, for instance particle acceleration and shocks caused by solar eruptions and flares (Kassim et al., 2010). Type III bursts are signatures of beams of energetic electrons traveling through the corona. One of the important signatures of shocks and shock acceleration are Type II bursts. Type IV bursts are attributed to electrons trapped in closed field lines in the post-flare arcades produced by solar flares (Aschwanden, 2005).

The antenna has two different polarization outputs (EastWest and North-South), which makes it possible to study also the polarization properties of solar flares.

This article gives an overview of the new solar monitoring system and the first solar radio burst that were observed with 
it, as well as plans for some future scientific work making use of its data.

\section{2 | TECHNICAL OVERVIEW}

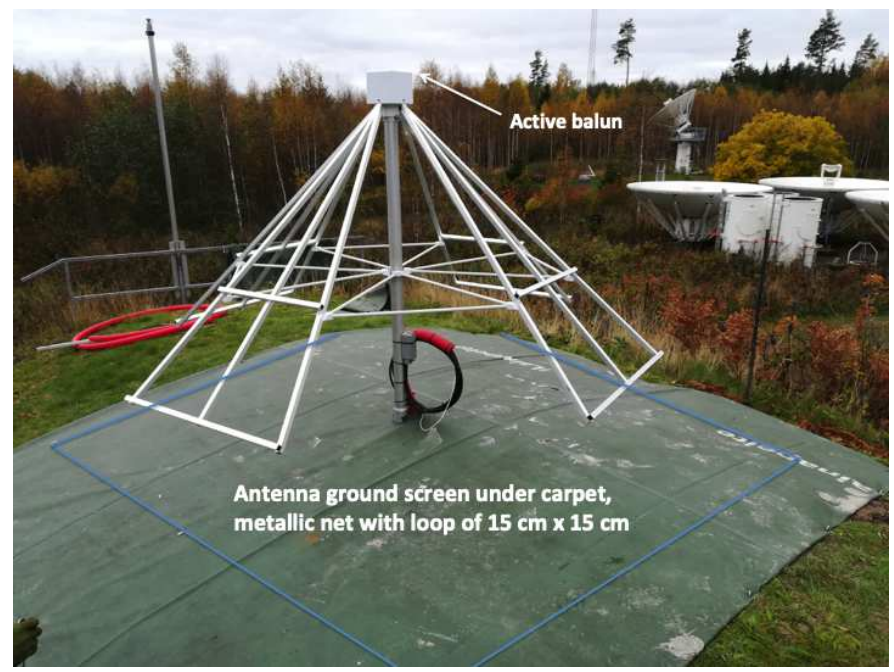

FIGURE 1 The METSOLA long wavelength array antenna installed in Metsähovi Radio Observatory.

An overview of the antenna installation is presented in Figure 1 The main parts of the system are: crossed dipole antenna (CDA), active balun on the top of the antenna, and metallic ground screen (under the green plastic carpet shown in Figure 1 . The metallic ground screen is for preventing back reflections and it consists of steel grid with $15-\mathrm{cm}$ cell size.

The antenna structure is made of aluminium frames and it does need a heavy foundation. The lightweight structure is simply attached to wedges hammered into the ground, and this is enough to withstand the most severe local weather; the first winter season 2017-2018 allowed testing the system in cold temperatures (down to $-25^{\circ} \mathrm{C}$ ), high wind speeds, and heavy snowfall.

The front end electronics in the antenna contains an active balun that converts the balanced dipole to unbalanced $50 \mathrm{ohm}$ impedance for connection to a coaxial cable transmission line and also provides an approximately $35 \mathrm{~dB}$ gain. The active balun is powered through coaxial cable ( $R G$ 214, $30 \mathrm{~m}$ in length) by a bias-tee arrangement. The detailed construction of the balun is included in Figure 2 The detailed documentation of the CDA and active balun can be found in Reeve (2018). The nominal attenuation of the coaxcial cable RG 214 at $70 \mathrm{MHz}$ is only $1.9 \mathrm{~dB}$ over $30 \mathrm{~m}$. The cables run in underground protection pipes all the way from the antenna to the control room.
The nominal upper frequency limit of the antenna is $90 \mathrm{MHz}$. We noticed, however, that the antenna has a rather good response up to $120 \mathrm{MHz}$ and we subsequently extended the observation range to $120 \mathrm{MHz}$.

\section{1 | Up-conversion}

The operational frequency range of the Callisto spectrometer starts at $45 \mathrm{MHz}$. Thus, if we want to observe and utilize the whole band of the spectrometer, a frequency up-conversion is needed. A simple up-converter, based on a voltage controlled oscillator, was designed and built for this purpose. In Figure 3 the layout of the up-conversion system is presented. It converts the detected RF-signal band, 5-120 MHz, to a higher band, 761.216-878.216 MHz. Only the upper side band is recorded. Currently, only one polarization $(\mathrm{N}-\mathrm{S})$ is up-converted; another up-converter will be added to the other polarization as well, after several other solar flares have been observed and when we are confident that the up-conversion system works as desired and that the overall performance is reasonably good.

\section{2 | Radio Frequency Interference - RFI}

One of the main problems when making radio astronomical observations at lower frequencies $(f \simeq 100 \mathrm{MHz}$ ) is radio frequency interference (RFI). This frequency range is allocated for various services such as broadcasting, amateur and mobile (Ficora, 2018). Upon investigating the local RFI environment at METSOLA operational frequencies, we found that the band between approximately 30 and $80 \mathrm{MHz}$ is still reasonably free from disturbances and usable for solar observations, while other adjoining bands are heavily contaminated. It should be noted that there are no allocations to the radio astronomy services inside of this band $(5-120 \mathrm{MHz})$. The detected signals from powerful solar flares are strong enough that they can be observed even if the environment is not fully clear of RFI and other unwanted signals. We are also prepared to add extra front-end filtering if the RFI environment deteriorates.

\section{3 | THE FIRST LIGHT - SOLAR RADIO BURST ON MARCH 302018}

The first solar radio flare was observed with METSOLA on March 30th, 2018. The event was classified as a GOES C4.6 flare. The origin of the flare was the NOAA active region 12703. The burst was detected in both polarizations, shown separately in two panels in Figure 4 . The flare was only detectable at the lower frequencies $(<100 \mathrm{MHz})$ observed with 


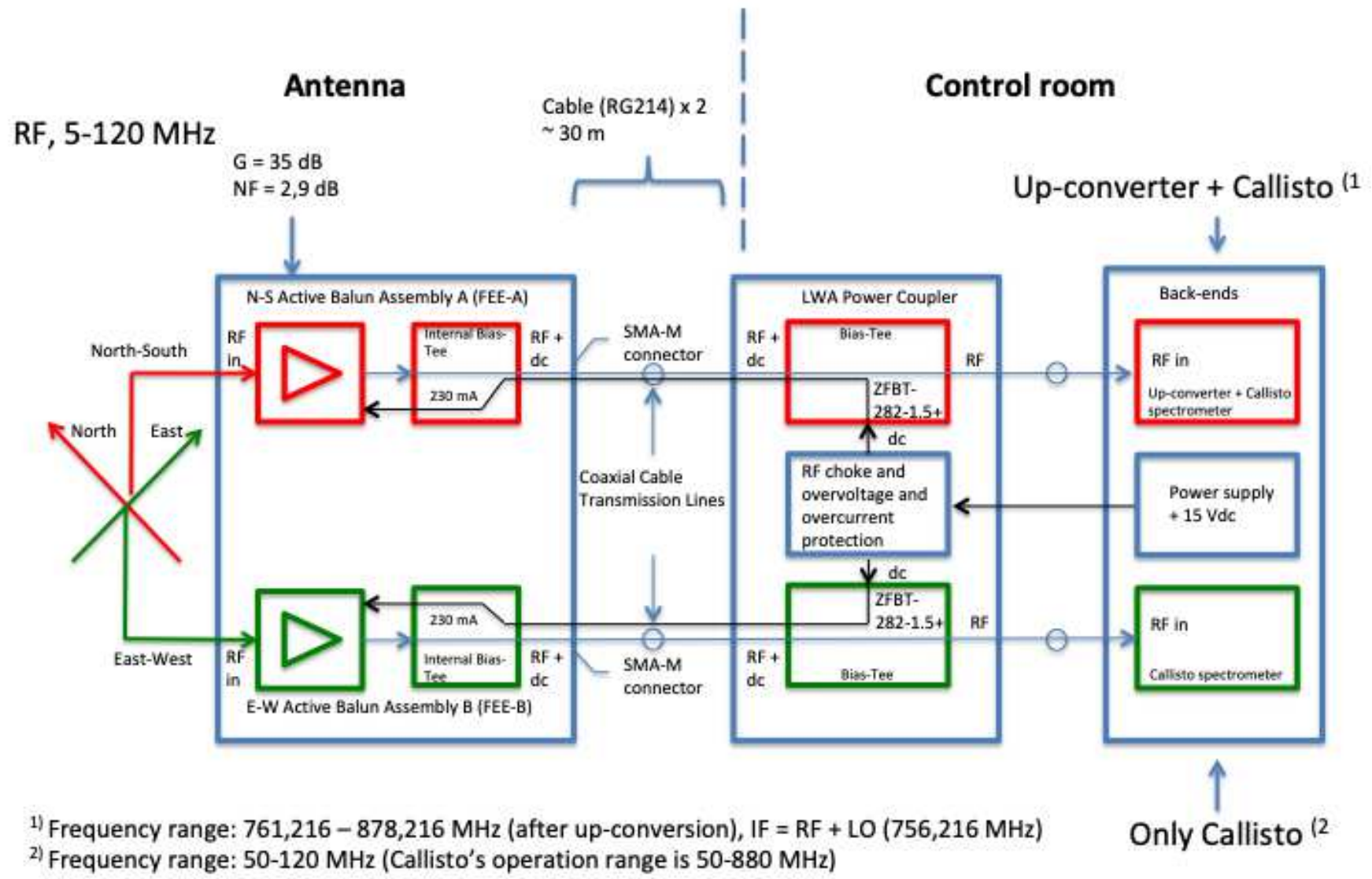

FIGURE 2 Schematics of the METSOLA system. The antenna part (left-hand side) contains the antenna and a balun. Spectrometers and the up-converter system are located in the control room. Adapted from Reeve (2018).

METSOLA. The other solar radio instruments at MRO, operated at higher frequencies, did not detect it. This highlights the added value of the low frequency solar radio observations.

The flare was of a short duration and had a rapid drift, which can be seen in Figure 4 thus it can be classified as a Type III burst. It is difficult to make any detailed analysis of the polarization properties of this particular flare on the basis of this observation. The burst properties (e.g., shape and duration) are not exactly the same at both polarizations, but more data are needed to fully verify the behaviour of the two polariation channels of the system.

\subsection{Drift speeds and electron densities of the detected solar flare}

It is possible to estimate the drift rate, the electron density and even the height propagation properties of the solar radio burst on the basis of the observed dynamic spectra. The frequency drift rate of the solar radio burst can be estimated as (Pohjolainen et al., 2007)

$$
\frac{\mathrm{d} f}{\mathrm{~d} t}=\frac{f_{0}-f_{1}}{t_{0}-t_{1}}
$$

where $f_{0}$ and $f_{1}$ are the frequency of the flare in start $\left(t_{0}\right)$ and end $\left(t_{1}\right)$ of the observerd flare, respectively.

In the left-hand panel (N-S polarization) of Figure 4 straight lines mark the start and end of the observed flare: $f_{0}$ is $64 \mathrm{MHz}, f_{1}$ is $50 \mathrm{MHz}$ and $t_{0}-t_{1}$ is 3 seconds, giving drift rate $4.7 \mathrm{MHz} / \mathrm{s}$. Also the other observed flares (E-W polarization in Figure 4 yield similar drift rates. The result matches with the earlier observation, e.g. Melnik et al. (2015), where observed drift rates varied between $0.44 \mathrm{MHz} / \mathrm{s}$ and $12 \mathrm{MHz} / \mathrm{s}$ for Type III solar bursts.

The connection between the plasma frequency $\left(v_{\text {pe }}\right)$ and the electron density $\left(n_{\mathrm{e}}\right)$ can be estimated as (Bastian et al., 1998; 


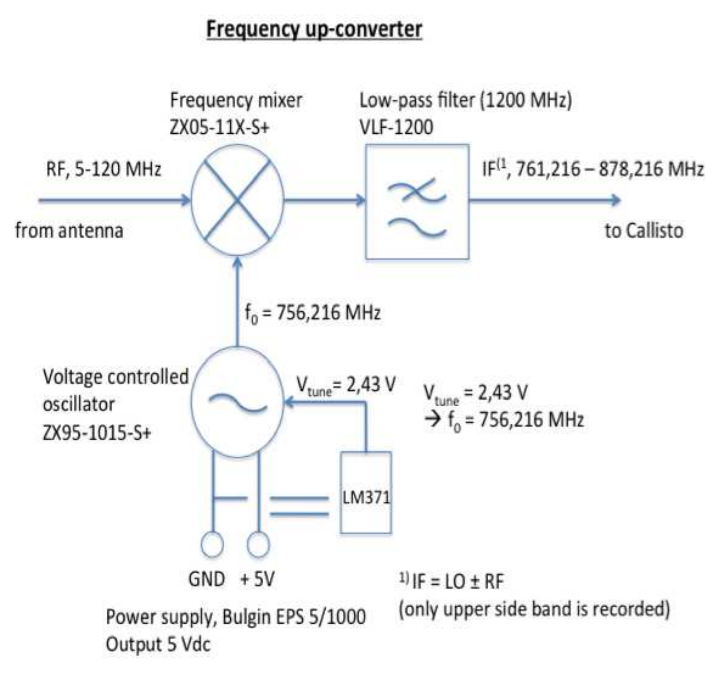

FIGURE 3 The block diagram of the self-made up-converter used for the METSOLA system. The up-converter system consist of a voltage-controller oscillator and a stable voltage source, mixer and low-pass filter.

Pohjolainen et al., 2007)

$$
v_{\mathrm{pe}} \approx 9000 \times \sqrt{n_{e}},
$$

leading to electron densities of $3 \times 10^{7} \mathrm{~cm}^{-1}$ and $5 \times 10^{7} \mathrm{~cm}^{-1}$, for $50 \mathrm{MHz}$ and $64 \mathrm{MHz}$, respectively.

The height propagation can be estimated, for instance, using the Newkirk 1st-order atmospheric density model (Li et al., 2009; Wijesekera et al., 2018)

$$
n_{\mathrm{e}}=n_{0} \times 10^{4.32 \frac{R_{\odot}}{R}}
$$

where $n_{0}$ is $4.2 \times 10^{4} \mathrm{~cm}^{-1}, R$ is distance from the solar center and $R_{\odot}$ is the solar radius. This gives estimates for the he propagation height of the solar flare at $50 \mathrm{MHz}$ and $64 \mathrm{MHz}$ as $1.5 R_{\odot}$ and $1.4 R_{\odot}$, respectively, placing the observed event in the solar corona, as expected.

\section{4 | FUTURE WORKS}

\section{1 | Technical upgrades}

Adding a continuous calibration capability to the system would be very useful for obtaining a more accurate estimate of the flux of the observed solar bursts. The implementation is not straightforward and remains to be resolved. Also, once we have confirmed that the use of the up-converter in one output channel of the current system works as expected, an up-converter will be added to the other polarization as well. Furthermore, we are experimenting with various approaches to develop automatic flare detection algorithms, in order to semi- or fully automatically distinguish solar-related signals from RFI that currently frequently trigger false "detections" at the METSOLA frequency range.

\section{2 । Scientific projects}

The primary scientific goals for exploiting METSOLA data include statistical analysis of Type II and III solar bursts, and multi-frequency analysis of individual flares.

We are planning to make statistical analysis of the drift rates of the Type II and III solar bursts for which we are now gathering data. Complementing them with Callisto data that date back to 2010 and are also being continuously updated, we will cover the frequency range $5-880 \mathrm{MHz}$. It is anticipated, however, that to be able to run a meaningful analysis on these data we will need to wait for some more years, for the solar activity to start rising again towards the next activity maximum.

Finally, we are looking forward to detect solar events simultaneously with different radio-frequency instruments, as MRO now has the capability to observe solar flares all the way from meter to millimeter wavelengths.

\section{5 | CONCLUSIONS}

We have presented a system that will be used for solar activity monitoring at low radio frequencies, $5-120 \mathrm{MHz}$, installed at the Aalto University Metsähovi Radio Observatory. It has a simple, weather resistant structure and low installation and maintenance costs.

Now that the Sun is entering a new activity cycle, the new instrument will be able to gather data all the way from the ostensibly quiescent Sun towards the more active phases. The data will be used to analyse characteristics of Type II and III solar bursts over a full solar cycle, as well as to study individual interesting events using an extensive range of multifrequency radio data obtained at $\mathrm{MRO}$ from $5 \mathrm{MHz}$ to $37 \mathrm{GHz}$.

\section{I ACKNOWLEDGEMENT}

The low wavelength antenna presented here is manufactured by Reeve Engineering, http://http://www.reeve.com.

The Callisto spectrometer is designed by Christian Monstein, http://http://www.e-callisto.org.

\section{REFERENCES}

Aschwanden, M. J. 2005, Pour la Science

Bastian, T. S., Benz, A. O., \& Gary, D. E. 1998, ARA\&A, 36, 131

e-Callisto, International Network of Solar Radio Spectrometers, a Space Weather Instrument Array http://www.e-callisto.org (24.11.2018) 

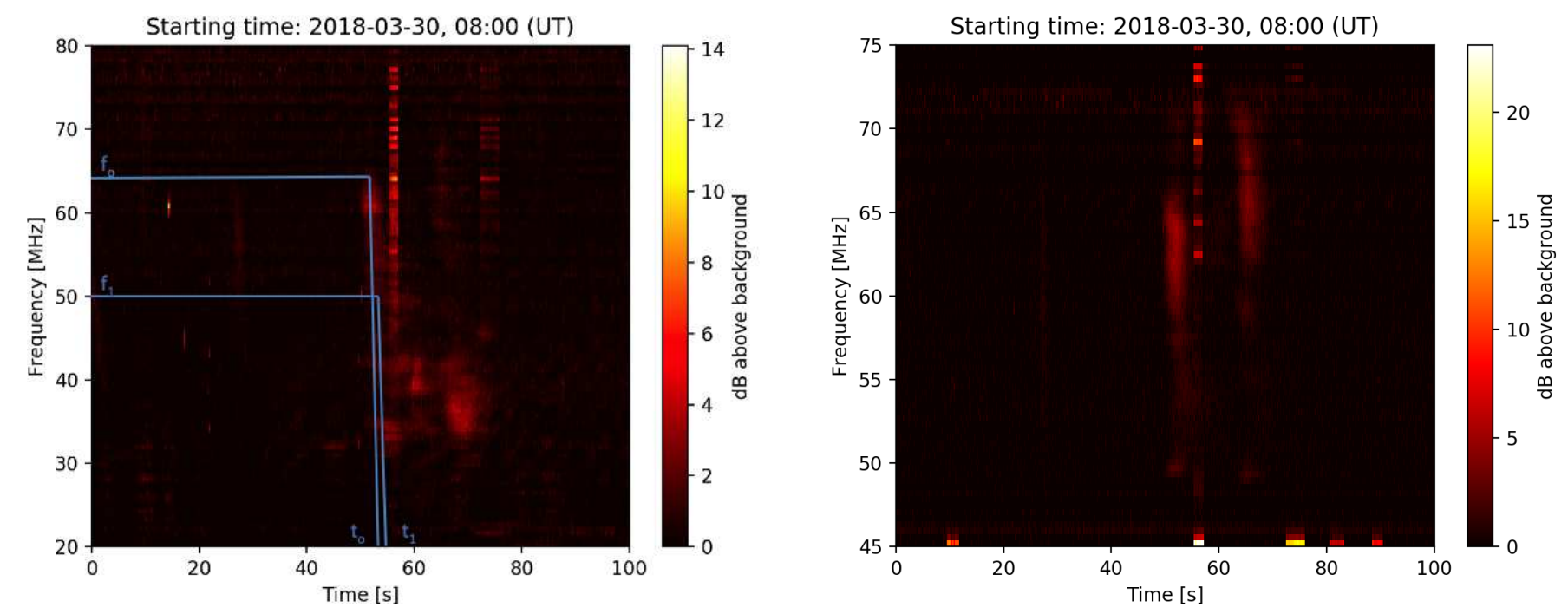

FIGURE 4 The first solar flare (Type III) observed with METSOLA. The left- and right-hand panels show N-S and E-W polarization, respectively. The intense, sharp-edged vertical line at $\sim 58$ seconds is caused by a RFI spike. The other distinguishable areas in the plot are solar-related activity. On the left-hand panel, start and end times are annoteted, with corresponding characteristic frequencies, as described in Sec. 3.1

FICORA's Radio Frequency Regulation - Frequency Allocation Table in Finland, https://www.viestintavirasto.fi/attachments/maaraykset/Taajuusjakotaulukko_englanti_3.1.2018.pdf (24.11.2018)

Hicks, B. C., Paravastu-Dalal, N., Stewart, K. P., et al. 2012, PASP, 124, 1090

Kallunki, J., Tornikoski, M., Tammi, J., et al. 2018, Astronomische Nachrichten, 339, 204

Kassim, N., White, S., Rodriquez, P., et al. 2010, Advanced Maui Optical and Space Surveillance Technologies Conference, E59

Li, B., Cairns, I. H., \& Robinson, P. A. 2009, Journal of Geophysical Research (Space Physics), 114, A02104

Mann, G., Breitling, F., Vocks, C., et al. 2018, A\&A, 611, A57

Melnik, V. N., Brazhenko, A. I., Konovalenko, A. A., et al. 2015, Sol. Phys., 290, 193

Pohjolainen, S., van Driel-Gesztelyi, L., Culhane, J. L., Manoharan, P. K., \& Elliott, H. A. 2007, Sol. Phys., 244, 167

Reeve Observatory, Anchorage, Alaska USA http://www.reeve.com/RadioScience/Antennas/ActiveCrossed-Dipole/LWA_Antenna.htm (24.11.2018)

J.V Wijesekera et al. 2018, J. Phys.: Conf. Ser. 1005012046

How cite this article: Kallunki J., M. Tornikoski, E. Oinaskallio, P. Kirves J. Aatrokoski A. Mujunen, and J. Tammi (2018), Properties of low frequency radio solar radio observations

How cite this article: Kallunki J., M. Tornikoski, E. Oinaskallio, P. Kirves J. Aatrokoski A. Mujunen, and J. Tammi (2018), Properties of low frequency radio solar radio observations 